\title{
The Effect of Three-Dimensional Freestream Disturbances on the Supersonic Flow Past a Wedge
}

Peter W. Duck

D. Glenn Lasseigne

Old Dominion University, dlasseig@odu.edu

M. Y. Hussaini

Follow this and additional works at: https://digitalcommons.odu.edu/mathstat_fac_pubs

Part of the Applied Mathematics Commons, Fluid Dynamics Commons, and the Plasma and Beam Physics Commons

\section{Repository Citation}

Duck, Peter W.; Lasseigne, D. Glenn; and Hussaini, M. Y., "The Effect of Three-Dimensional Freestream Disturbances on the Supersonic Flow Past a Wedge" (1997). Mathematics \& Statistics Faculty Publications. 32.

https://digitalcommons.odu.edu/mathstat_fac_pubs/32

\section{Original Publication Citation}

Duck, P. W., Lasseigne, D. G., \& Hussaini, M. Y. (1997). The effect of three-dimensional freestream disturbances on the supersonic flow past a wedge. Physics of Fluids, 9(2), 456-467. doi:10.1063/1.869140

This Article is brought to you for free and open access by the Mathematics \& Statistics at ODU Digital Commons. It has been accepted for inclusion in Mathematics \& Statistics Faculty Publications by an authorized administrator of ODU Digital Commons. For more information, please contact digitalcommons@odu.edu. 
The effect of three-dimensional freestream disturbances on the supersonic flow past a wedge

Peter W. Duck, D. Glenn Lasseigne, and M. Y. Hussaini

Citation: Physics of Fluids 9, 456 (1997); doi: 10.1063/1.869140

View online: http://dx.doi.org/10.1063/1.869140

View Table of Contents: http://aip.scitation.org/toc/phf/9/2

Published by the American Institute of Physics

\section{Articles you may be interested in}

Interaction of Linear Waves with Oblique Shock Waves

The Physics of Fluids 11, 2350 (2003); 10.1063/1.1691825

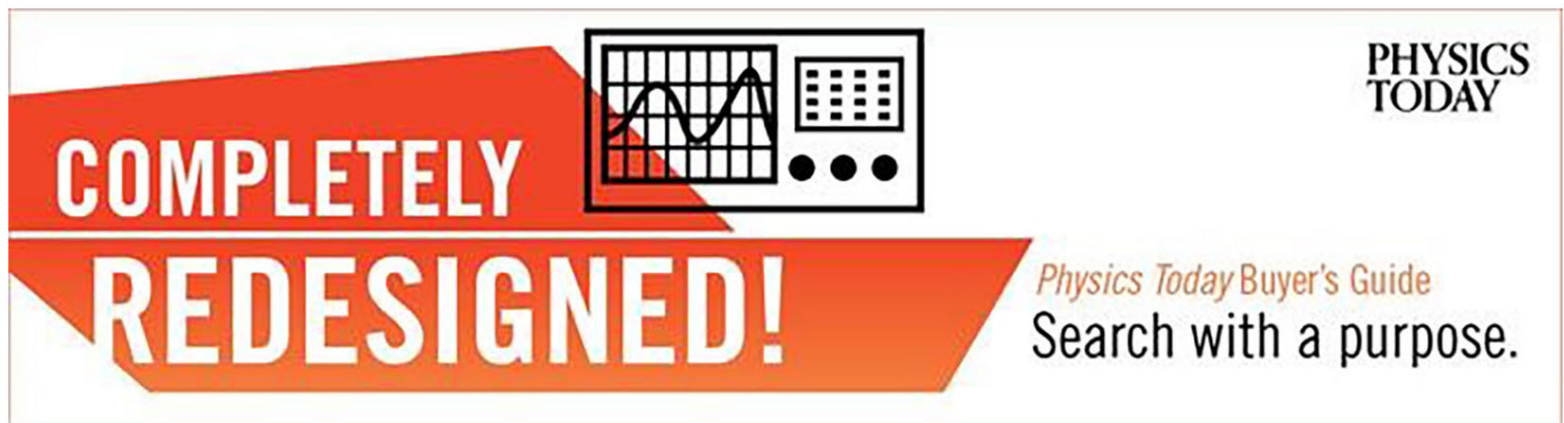




\title{
The effect of three-dimensional freestream disturbances on the supersonic flow past a wedge
}

\author{
Peter W. Duck \\ Department of Mathematics, University of Manchester, Manchester M13 9PL, United Kingdom \\ D. Glenn Lasseigne \\ Department of Mathematics and Statistics, Old Dominion University, Norfolk, Virginia 23529 \\ M. Y. Hussaini \\ Program in Computational Science and Engineering, Florida State University, Tallahassee, Florida 32306
}

(Received 10 May 1996; accepted 30 September 1996)

\begin{abstract}
The interaction between a shock wave (attached to a wedge) and small amplitude, three-dimensional disturbances of a uniform, supersonic, freestream flow are investigated. The paper extends the two-dimensional study of Duck et al. [P W. Duck, D. G. Lasseigne, and M. Y. Hussaini, "On the interaction between the shock wave attached to a wedge and freestream disturbances," Theor. Comput. Fluid Dyn. 7, 119 (1995) (also ICASE Report No. 93-61)] through the use of vector potentials, which render the problem tractable by the same techniques as in the two-dimensional case, in particular by expansion of the solution by means of a Fourier-Bessel series, in appropriately chosen coordinates. Results are presented for specific classes of freestream disturbances, and the study shows conclusively that the shock is stable to all classes of disturbances (i.e., time periodic perturbations to the shock do not grow downstream), provided the flow downstream of the shock is supersonic (loosely corresponding to the weak shock solution). This is shown from our numerical results and also by asymptotic analysis of the Fourier-Bessel series, valid far downstream of the shock. (C) 1997 American Institute of Physics. [S1070-6631(97)02701-3]
\end{abstract}

\section{INTRODUCTION}

The interaction of freestream disturbances with shock waves is an important practical problem, with particular regard to the operation of high-speed flight vehicles. Of particular interest are the consequences of these disturbances on boundary-layer receptivity, and this paper may be regarded as a step in this process.

In the case of an isolated shock, Moore, ${ }^{1}$ Ribner $^{2}$ and McKenzie and Westphal ${ }^{3}$ showed that a single wavelength entropy, acoustic or vorticity wave, upstream of the shock, produces a disturbance comprising a mixture of all three classes downstream of the shock. When the shock is associated with the flow past a rigid body, the situation is much more complicated, due to the reflection and refraction of disturbances between the body surface and the shock itself, the latter distorting as a result, a process which further complicates the flow structure. This process was considered by Carrier $^{4}$ and Van Dyke ${ }^{5}$ with particular regard to the problem of supersonic flow past a wedge performing small amplitude oscillations, the shock remaining attached to the wedge tip. These problems raise questions regarding the stability of the shock, and this aspect has been considered in the twodimensional context (associated with wedge flows, the shock remaining attached to the wedge tip at all times) by Levinson, ${ }^{6}$ Carrier, ${ }^{7}$ Henderson and Atkinson, ${ }^{8}$ Rusanov and Sharakshannae ${ }^{9}$ and Salas and Morgan. ${ }^{10}$ The overall conclusion is that if the flow downstream of the shock is subsonic (loosely classified as the strong shock solution), then the shock is unstable, in so far as disturbances grow downstream. If, on the other hand, the flow behind the shock is supersonic (loosely classified as the weak shock solution), then the shock is stable, with disturbances not growing in amplitude downstream.

This aspect was considered in some detail by Duck et al., ${ }^{11}$ again for the case of supersonic flow past wedges, subject to two-dimensional disturbances. This latter paper (where there is also a more detailed summary of previous work in this area) showed that two-dimensional disturbances, impinging upon the weak shock solution, do not grow downstream, but rather produce constant amplitude or weakly decaying oscillatory waves downstream. The conditions which distinguish between the two types of behaviours were determined and a decomposition of the far-field, suggested by the analytic solution, was made. The result was that there is a shadow region dependent on the incoming disturbance. If the direction of propagation of the plane-wave acoustic disturbance generated at the shock by the plane-wave incoming disturbance intersects the wedge surface, then there is a nondecaying oscillatory pressure disturbance on the wedge surface. In any case, there are two weakly decaying singlewavelength oscillatory disturbances on the wedge surface that are due to the requirement that the shock remain attached. One is traveling at the mean-flow speed plus the speed of sound, and the other travels at the mean-flow speed minus the speed of sound. Thus these are clearly regular acoustic waves which emanate from a point source (the wedge apex) of fixed temporal frequency as prescribed by the incoming disturbance. In addition to these more obvious effects, there is also a local field effect which is broad based in wavelength. While the first three modes of pressure disturbances at the wedge surface can indeed interact with the boundary layer, it is the last disturbance with the inclusion of short wavelength disturbances that should have the most ef- 
fect on the growing boundary layer. In this latter paper, the side issue of shock stability to two-dimensional disturbances was also addressed. The aim of the present paper is to extend the ideas of Duck et al. ${ }^{11}$ to three-dimensional disturbances; indeed, little three-dimensional work of this type appears to have been undertaken in the past.

\section{FORMULATION}

Throughout this paper, we use subscript 1 to denote quantities upstream of the shock, and subscript 2 to denote downstream quantities. The wedge is taken to make an angle $\theta$ with respect to the oncoming flow, with $\left(x^{*}, y^{*}, z^{*}\right)$ coordinates parallel and perpendicular to the upstream flow, with $z^{*}$ being the "crossflow" direction. The upstream basic flow velocity has magnitude $U_{1}^{*}$, with Mach number $M_{1}$ and density $\rho_{1}^{*}$, and temperature $T_{1}^{*}$. The ratio of specific heats $\gamma$ is assumed to be constant, and throughout this paper all our numerical results take $\gamma=1.4$. The velocity vector is written as $U_{1}^{*}(u, v, w)$, with respect to $\left(x^{*}, y^{*}, z^{*}\right)$ coordinates, and the density is then written $\rho_{1}^{*} \rho$, pressure as $\rho_{1}^{*} R^{*} T_{1}^{*} p$ (where $R^{*}$ denotes the gas constant) and the temperature is $T_{1}^{*} T$.

The Rankine-Hugoniot relations provide a link between conditions upstream and downstream of the shock. For the basic (steady) flow, when the downstream flow is uniform and parallel to the wedge surface, the following classical result is obtained (see, for example, Liepmann and Roshko $^{12}$ ):

$$
\frac{\tan (\beta-\theta)}{\tan \beta}=\frac{\overline{u_{2}}}{\overline{u_{1}}}=\frac{\rho_{1}}{\rho_{2}}=\frac{(\gamma-1) M_{1}^{2} \sin ^{2} \beta+2}{(\gamma+1) M_{1}^{2} \sin ^{2} \beta},
$$

where $\beta$ is the angle between the shock and the wedge centerline (i.e., $y^{*}=0$ ) and $\overline{u_{1}}$ and $\overline{u_{2}}$ are the non-dimensional velocity components perpendicular to the shock.

The relationship (1) yields two possible values for $\beta$, for a given value of $\theta$ (for $\theta<\theta_{\max }\left(M_{1}\right)$ ) with the so-called "weak shock" solution generally being characterized by supersonic flow downstream of the shock, whilst the other "strong shock" solution is generally characterized by downstream subsonic flow. Note, however, that there does exist a small, weak shock regime, close to $\theta=\theta_{\max }$ where the downstream flow is subsonic, i.e., the downstream sonic line does not quite coincide with $\theta_{\max }$.

We shall be concerned with the effect of small amplitude disturbances, which are introduced into the flow ahead of the shock. The corresponding two-dimensional results, as studied by McKenzie and Westphal, ${ }^{3}$ for example, may be extended into the three-dimensional context, in quite a straightforward manner, when the $z^{*}$ variation is taken to be periodic (although other, more general $z^{*}$ variations could be accomplished using Fourier transforms). We take $\varepsilon(\ll 1)$ to be a measure of the amplitude of the freestream disturbance, and it then turns out that the upstream disturbance may be classified into three distinct classes, just as in the twodimensional case.

(i) Acoustic waves: these are characterized by having a pressure perturbation, with corresponding perturbations in velocity, density and temperature, but no change in entropy or vorticity, to $O(\varepsilon)$. Disturbances of this class take the form

$$
\begin{aligned}
& p=1+\hat{E}+O\left(\varepsilon^{2}\right), \\
& u=1-\frac{\alpha_{1} \varepsilon \hat{E}}{\gamma M_{1}^{2}\left(\alpha_{1}+\omega\right)}+O\left(\varepsilon^{2}\right), \\
& v=-\frac{\alpha_{2} \varepsilon \hat{E}}{\gamma M_{1}^{2}\left(\alpha_{1}+\omega\right)}+O\left(\varepsilon^{2}\right), \\
& w=-\frac{\alpha_{3} \varepsilon \hat{E}}{\gamma M_{1}^{2}\left(\alpha_{1}+\omega\right)}+O\left(\varepsilon^{2}\right), \\
& \rho=1+\frac{\varepsilon \hat{E}}{\gamma}+O\left(\varepsilon^{2}\right),
\end{aligned}
$$

and

$$
T=1-\frac{\varepsilon(\gamma-1) \hat{E}}{\gamma}+O\left(\varepsilon^{2}\right),
$$

where $\hat{E}$ is the normal mode exponential, i.e.,

$$
\hat{E}=\exp \left\{i \alpha_{1} x_{1}+i \alpha_{2} y_{1}+i \alpha_{3} z+i \omega t\right\},
$$

with

$$
\omega=-\alpha_{1} \pm \frac{1}{M_{1}}\left[\alpha_{1}^{2}+\alpha_{2}^{2}+\alpha_{3}^{2}\right]^{1 / 2}
$$

being the frequency for the given wave numbers. In the above, $x_{1}$ and $y_{1}$ are parallel and perpendicular to the upstream flow, respectively (both perpendicular to $z$ ); the nondimensionalization is carried out using one of the wavelengths of the disturbances as the typical length scale, e.g., by setting $\alpha_{1}$ to unity. The modes with the positive sign above in (9) are usually referred to as the slow modes, whilst those with the negative sign are usually referred to as the fast modes.

(ii) Vorticity waves: these are characterized by having no density, temperature or pressure disturbances to $O(\varepsilon)$, and thus the upstream flow takes the form

$$
\begin{aligned}
& u=1+\varepsilon \Omega_{z} \frac{\alpha_{2}}{\alpha_{1}^{2}+\alpha_{2}^{2}} \hat{E}+O\left(\varepsilon^{2}\right), \\
& v=\varepsilon\left[-\Omega_{z} \frac{\alpha_{1}}{\alpha_{1}^{2}+\alpha_{2}^{2}}-\Omega_{x} \frac{\alpha_{3}}{\alpha_{2}^{2}+\alpha_{3}^{2}}\right] \hat{E}+O\left(\varepsilon^{2}\right), \\
& w=\varepsilon \Omega_{x} \frac{\alpha_{2}}{\alpha_{2}^{2}+\alpha_{3}^{2}} \hat{E}+O\left(\varepsilon^{2}\right)
\end{aligned}
$$

and

$$
p, T, \rho=1+O\left(\varepsilon^{2}\right),
$$

with

$$
\omega=-\alpha_{1} .
$$

The exponential term $\hat{E}$ is unaltered from (8), and the parameters $\Omega_{z}$ and $\Omega_{x}$ must be specified. In comparison with the two-dimensional case, the three-dimensional case allows for an extra vorticity mode. 
(iii) Entropy waves: these have no disturbance pressure or velocity components to $O(\varepsilon)$, and may therefore be written in the form

$$
\begin{aligned}
& \rho=1+\varepsilon \hat{E}+O\left(\varepsilon^{2}\right), \\
& T=1-\varepsilon \hat{E}+O\left(\varepsilon^{2}\right), \\
& u, p=1+O\left(\varepsilon^{2}\right),
\end{aligned}
$$

and

$$
v, w=O\left(\varepsilon^{2}\right),
$$

with $\omega$ defined by (14) and $\hat{E}$ by (8), again.

In the following section of the paper we consider the effects that these waves have on a shock wave attached to a wedge. The complication introduced by the presence of the wedge is that a single mode of the above type, upstream of the shock, will trigger modes of all three types, of all wave numbers behind the shock; the only quantities preserved across the shock (provided $\varepsilon \ll 1$ ) are the frequency parameter $\omega$ and the spanwise wave number $\alpha_{3}$.

\section{THE DOWNSTREAM SOLUTION}

The flow downstream of the shock comprises the uniform flow solution plus a small amplitude $(O(\varepsilon))$ perturbation, triggered by the freestream disturbances described in the previous section. We take non-dimensional coordinates parallel and perpendicular to the wedge (perpendicular to the $z$ direction in both cases) as $x_{2}$ and $y_{2}$, respectively, with corresponding velocity components $\left(u_{2}, v_{2}\right)$.

We now write the solution downstream of the shock in the form

$$
\begin{aligned}
& u_{2}=U_{2}+\varepsilon \widetilde{u}+O\left(\varepsilon^{2}\right), \\
& v_{2}=\varepsilon \widetilde{v}+O\left(\varepsilon^{2}\right), \\
& w=\varepsilon \widetilde{w}+O\left(\varepsilon^{2}\right), \\
& \rho=\rho_{2}+\varepsilon \widetilde{\rho}+O\left(\varepsilon^{2}\right), \\
& p=p_{2}+\varepsilon \widetilde{p}+O\left(\varepsilon^{2}\right),
\end{aligned}
$$

and

$$
T=T_{2}+\varepsilon \widetilde{T}+O\left(\varepsilon^{2}\right) .
$$

Substitution of these expansions into the governing (inviscid) equations of motion, continuity and energy equations and equations of state then leads to

$$
\begin{aligned}
& \widetilde{\rho_{t}}+U_{2}{\widetilde{\rho_{x_{2}}}}+\rho_{2}{\widetilde{u_{x_{2}}}}+\rho_{2}{\widetilde{v_{y_{2}}}}+\rho_{2} \widetilde{w}_{z}=0, \\
& \rho_{2}\left\{\tilde{u_{t}}+U_{2} \tilde{u_{x_{2}}}\right\}+\frac{1}{\gamma M_{1}^{2}} \widetilde{p}_{x_{2}}=0, \\
& \rho_{2}\left\{\widetilde{v_{t}}+U_{2} \widetilde{v_{x_{2}}}\right\}+\frac{1}{\gamma M_{1}^{2}} \widetilde{p_{y_{2}}}=0, \\
& \rho_{2}\left\{\widetilde{w}_{t}+U_{2} \widetilde{w}_{x_{2}}\right\}+\frac{1}{\gamma M_{1}^{2}} \widetilde{p_{z}}=0, \\
& \rho_{2}\left\{\widetilde{T}_{t}+U_{2} \widetilde{T}_{x_{2}}\right\}-\frac{\gamma-1}{\gamma}\left\{\widetilde{p_{t}}+U_{2}{\widetilde{p_{x}}}_{2}\right\}=0,
\end{aligned}
$$

and

$$
\widetilde{p}=\rho_{2} \widetilde{T}+T_{2} \widetilde{\rho} .
$$

Much of the success of the approach used by Carrier, ${ }^{4}$ Van Dyke ${ }^{5}$ and indeed by Duck et al. ${ }^{11}$ was due to the ability of being able to split the solution into two components, the one including a velocity potential (which represented the acoustic wave component of the flow), and the other a stream function (which represented the vorticity wave component of the flow). It is clearly not possible to use the latter in the context of three-dimensional flows, however, an alternative is the introduction of a vector potential. This concept, originally due to Poincare,${ }^{13}$ has been used in a number of fluid mechanics investigations over the years, although the appropriate boundary conditions have been the subject of discussion, as detailed by Aziz and Hellums. ${ }^{14}$

Specifically we write the perturbation velocity vector $\widetilde{\mathbf{u}}=(\widetilde{u}, \widetilde{v}, \widetilde{w})$ in the form

$$
\widetilde{\mathbf{u}}=\boldsymbol{\nabla} \phi+\boldsymbol{\nabla} \wedge \mathbf{E},
$$

where $\phi$ is the velocity potential, and $\mathbf{E}$ the vector potential. The above constitutes a non-unique representation for $\widetilde{\mathbf{u}}$, and as such an additional relationship may be (arbitrarily) specified. The most popular choice, and the one that we pursue here, is that the vector potential can be required to be solenoidal, i.e.,

$$
\boldsymbol{\nabla} \cdot \mathbf{E}=0 .
$$

This leads to the vorticity vector then being merely the quantity $-\nabla^{2} \mathbf{E}$. Substitution of (31) into (26)-(28) leads to the equations

$$
\nabla^{2}\left\{\phi_{t}+U_{2} \phi_{x_{2}}+\frac{p}{\gamma M_{1}^{2} \rho_{2}}\right\}=0,
$$

and

$$
\nabla^{2}\left\{\mathbf{E}_{t}+U_{2} \mathbf{E}_{x_{2}}\right\}=0 .
$$

It then follows that $\tilde{p}$ satisfies

$$
\tilde{p}=-\frac{\gamma p_{2}}{a_{s}^{2}}\left[\phi_{t}+U_{2} \phi_{x_{2}}\right],
$$

and elimination of the dependent variables $\widetilde{\rho}$ and $\widetilde{T}$ gives the equation for $\phi$

$$
\nabla^{2} \phi=\frac{1}{a_{s}^{2}}\left[\phi_{t t}+2 U_{2} \phi_{x t}+U_{2}^{2} \phi_{x_{2} x_{2}}\right],
$$

where

$$
a_{s}=\frac{T_{2}^{1 / 2}}{M_{1}} .
$$

If we write $\mathbf{E}=\left(E^{(1)}, E^{(2)}, E^{(3)}\right)$, then on account of our comments above, we impose the condition

$$
E_{x_{2}}^{(1)}+E_{y_{2}}^{(2)}+E_{z}^{(3)}=0 .
$$

A further quantity must also be introduced, namely $\psi^{\prime}\left(y_{2}, z, t\right)$, which describes the displacement of the shock wave from its undisturbed state. 
This type of formulation then allows us to follow closely the two-dimensional approach adopted by Duck et al. ${ }^{11}$ It is now possible to write the general solution for $\phi, \psi^{\prime}$, and the $E^{(n)}$ in the following form, assuming boundedness at the apex of the wedge:

$$
\begin{aligned}
\phi= & \exp \left(i \omega t-\frac{i \omega U_{2} x_{2}}{U_{2}^{2}-a_{s}^{2}}+i \alpha_{3} z\right) \\
& \times \sum_{\nu=0}^{\infty}\left\{a_{\nu} \cosh \left(\nu \theta_{2}\right)+b_{\nu} \sinh \left(\nu \theta_{2}\right)\right\} J_{\nu}\left(\hat{k}_{e} r\right), \\
E^{(n)}= & \exp \left(i \omega t-\frac{i \omega x_{2}}{U_{2}}-\frac{i \omega \lambda y_{2} a_{s}^{2}}{U_{2}\left(U_{2}^{2}-a_{s}^{2}\right)}+i \alpha_{3} z\right) \\
& \times \sum_{\nu=0}^{\infty} c_{\nu}^{(n)} J_{\nu}\left(\hat{k}_{e} \hat{\xi} y_{2}\right)
\end{aligned}
$$

and

$$
\psi^{\prime}=\exp \left(i \omega t-\frac{i \omega \lambda y_{2} U_{2}}{U_{2}^{2}-a_{s}^{2}}+i \alpha_{3} z\right) \sum_{\nu=0}^{\infty} d_{\nu} J_{\nu}\left(\hat{k} \hat{\xi}_{e} \hat{\xi} y_{2}\right),
$$

where we have written

$$
\begin{aligned}
& \hat{\omega}=\frac{\omega}{a_{s}}, \\
& \hat{k}=\frac{\hat{\omega}}{\hat{\beta}^{2}},
\end{aligned}
$$

$$
\begin{aligned}
& \hat{\beta}^{2}=\frac{U_{2}^{2}-a_{s}^{2}}{a_{s}^{2}}, \\
& \hat{k}_{e}^{2}=\hat{k}^{2}+\frac{\alpha_{3}^{2}}{\hat{\beta}^{2}}, \\
& \lambda=\cot (\beta-\theta), \\
& \hat{\xi}=\sqrt{\lambda^{2}-\hat{\beta}^{2}}, \\
& r^{2}=x_{1}^{2}-\hat{\beta}^{2} y_{2}^{2},
\end{aligned}
$$

and

$$
\tanh \theta_{2}=\hat{\beta} y_{2} / x_{2} .
$$

If we impose impermeability on the wedge surface, we immediately require

$$
b_{\nu}=0 \forall \nu,
$$

whilst if the shock remains attached at the tip, then $d_{0}=0$. As noted by Duck et al. ${ }^{11}$ (for example), it is also reasonable to set the $\nu=0$ coefficients of the other terms in the series to zero, which correspond to transient-type modes, and so we write

$$
a_{0}=c_{0}^{(1)}=c_{0}^{(2)}=c_{0}^{(3)}=0 .
$$

The key results for the velocity components and pressure are then

$$
\begin{aligned}
\tilde{u}= & -\exp \left(i \omega t-\frac{i U_{2} \omega x_{2}}{U_{1}^{2}-a_{s}^{2}}+i \alpha_{3} z\right) \sum_{\nu=0}^{\infty} \frac{\hat{k}_{e}}{2}\left[\frac{2 i \hat{\omega} U_{2}}{\hat{k}_{e} \hat{\beta}^{2} a_{s}} a_{\nu}+a_{\nu-1}\left(1+\delta_{\nu-1,0}\right)-a_{\nu+1}\right] \cosh \nu \theta_{2} J_{\nu}\left(\hat{k}_{e} r\right) \\
& +\exp \left(i \omega t-\frac{i \omega x_{2}}{U_{2}}-\frac{i \omega \lambda y_{2} a_{s}^{2}}{U_{2}\left(U_{2}^{2}-a_{s}^{2}\right)}+i \alpha_{3} z\right) \sum_{\nu=0}^{\infty}\left[\frac{\hat{k}_{e}}{2}\left(-\frac{2 i \lambda \hat{\omega} a_{s}}{\hat{k}_{e} U_{2} \hat{\beta}^{2}} c_{\nu}^{(3)}+\hat{\xi}\left(c_{\nu+1}^{(3)}-c_{\nu-1}^{(3)}\left(1+\delta_{\nu-1,0}\right)\right)\right)-i \widetilde{\beta} c_{\nu}^{(2)}\right] J_{\nu}\left(\hat{k}_{e} \hat{\xi} y_{2}\right),
\end{aligned}
$$

$$
\begin{aligned}
\widetilde{v}= & \exp \left(i \omega t-\frac{i U_{2} \omega x_{2}}{U_{1}^{2}-a_{s}^{2}}+i \alpha_{3} z\right) \sum_{\nu=0}^{\infty} \frac{\hat{k}_{e} \hat{\beta}}{2}\left(a_{\nu-1}+a_{\nu+1}\right) \sinh \left(\nu \theta_{2}\right) J_{\nu}\left(\hat{k}_{e} r\right) \\
& +\exp \left(i \omega t-\frac{i \omega x_{2}}{U_{2}}-\frac{i \omega \lambda y a_{s}^{2}}{U_{2}\left(U_{2}^{2}-a_{s}^{2}\right)}+i \alpha_{3} z\right) \sum_{z=0}^{\infty}\left[i \widetilde{\beta} c_{\nu}^{(1)}+\frac{i \omega}{U_{2}} c_{\nu}^{(3)}\right] J_{\nu}\left(\hat{k}_{e} \hat{\xi} y_{2}\right), \\
\widetilde{w}= & \left.\exp \left(i \omega t-\frac{i U_{2} \omega x_{2}}{U_{2}^{2}-a_{s}^{2}}+i \alpha_{3} z\right) \sum_{\nu=0}^{\infty} i \widetilde{\beta} a_{\nu} J_{\nu}\left(\hat{k}_{e} r\right) \cosh \nu \theta_{2}-\exp i \omega t+i \alpha_{3} z-\frac{i \omega x_{2}}{U_{2}}-\frac{i \omega \lambda y_{2} a_{s}^{2}}{U_{2}\left(U_{2}^{2}-a_{s}^{2}\right)}\right) \\
& \times \sum_{\nu=0}^{\infty}\left[\frac{i \hat{\omega} a_{s}}{U_{2}} c_{\nu}^{(2)}+\frac{\hat{k}_{e}}{2}\left(-\frac{2 i \lambda \hat{\omega} a_{s}}{\hat{k}_{e} U_{2} \hat{\beta}^{2}} c_{\nu}^{(1)}+\hat{\xi}\left(c_{\nu+1}^{(1)}-c_{\nu-1}^{(1)}\left(1+\delta_{\nu-1,0}\right)\right)\right)\right] J_{\nu}\left(\hat{k}_{e} \hat{\xi} y_{2}\right),
\end{aligned}
$$

and

$\tilde{p}=\frac{\gamma p_{2} \hat{k}_{e}}{2 a_{s}} \exp \left(i \omega t-\frac{i U_{2} \omega x_{2}}{U_{2}^{2}-a_{s}^{2}}+i \alpha_{3} z\right) \sum_{\nu=0}^{\nu=\infty}\left[\frac{2 i \hat{\omega}}{\hat{k}_{e} \hat{\beta}^{2}} a_{\nu}+\frac{U_{2}}{a_{s}} a_{\nu-1}\left(1+\delta_{\nu-1,0}\right)-\frac{U_{2}}{a_{s}} a_{\nu+1}\right] \cosh \left(\nu \theta_{2}\right) J_{\nu}\left(\hat{k}_{e} r\right)$, 
where $a_{-1}=c_{-1}^{(n)}=d_{-1}=0$, and where $\delta_{n, m}$ is the Kronecker delta.

Although the solutions above satisfy the impermeability and apex conditions, as yet these solutions do not satisfy the unsteady Rankine-Hugoniot conditions (McKenzie and Westphal ${ }^{3}$ ) on the shock; following (and extending) the results of Duck et al., ${ }^{11}$ these take the form

$$
\begin{aligned}
& u_{2}^{\prime}+\overline{u_{2}} \frac{\widetilde{\rho_{2}}}{\rho_{2}}=\left(1-\frac{1}{\rho_{2}}\right) R^{\prime}+\frac{1}{\rho_{2}}\left(\bar{u}, \widetilde{\rho_{1}}+u_{1}^{\prime}\right), \\
& u_{2}^{\prime}+\frac{1}{2} \overline{u_{2}} \frac{\widetilde{\rho_{2}}}{\rho_{2}}+\frac{a_{s}^{2}}{2 \gamma \overline{u_{2}}} \frac{\widetilde{p_{2}}}{p_{2}}=u_{1}^{\prime}+\frac{1}{2} \overline{u_{1}} \widetilde{\rho_{1}}+\frac{\widetilde{p_{1}}}{2 \gamma M_{1}^{2} \overline{u_{1}}}, \\
& u_{2}^{\prime}-\frac{a_{s}^{2}}{(\gamma-1) \overline{u_{2}}} \frac{\tilde{\rho_{2}}}{\rho_{2}}+\frac{a_{s}^{2}}{(\gamma-1) \overline{u_{2}}} \frac{\tilde{p_{2}}}{\overline{p_{2}}} \\
& =\left(1-\frac{\overline{u_{1}}}{\overline{u_{2}}}\right) R^{\prime}+\frac{\overline{u_{1}}}{\overline{u_{2}}} u_{1}^{\prime}-\frac{\tilde{\rho_{1}}}{(\gamma-1) M_{1}^{2} \overline{u_{2}}}+\frac{\widetilde{p_{1}}}{(\gamma-1) M_{1}^{2} \overline{u_{2}}},
\end{aligned}
$$

$v_{2}^{\prime}=v_{1}^{\prime}+\left(\overline{u_{1}}-\overline{u_{2}}\right) \psi_{\sigma}^{\prime}$,

and

$$
w_{2}^{\prime}=w_{1}^{\prime}+\left(\overline{u_{1}}-\overline{u_{2}}\right) \psi_{z}^{\prime},
$$

with

$$
R^{\prime}=\psi_{t}^{\prime}+\bar{v}_{1} \psi_{\sigma}^{\prime}
$$

In equations (56) $-(61), \sigma$ denotes the unperturbed distance along the shock, $\left(u^{\prime}, v^{\prime}, w^{\prime}\right)$ denote the velocity perturbations perpendicular to the undisturbed shock, parallel to the shock and perpendicular to the $z$ direction, and parallel to the $z$ direction, respectively, and $(\bar{u}, \bar{v}, 0)$ denote the corresponding base flow velocity components. Eliminating $\widetilde{\rho}_{2}$ from the above equations, and then utilizing (52)-(55), yields the following four equations for each of the $\nu \geqslant 0$ :

$$
\begin{aligned}
& \cos (\beta-\theta)\left\{-\frac{\hat{k}_{e}}{2}\left[\frac{2 i \hat{\omega} U_{2}}{\hat{k}_{e} \hat{\beta}^{2} a_{s}} a_{\nu}+a_{\nu-1}\left(1+\delta_{\nu-1,0}\right)-a_{\nu+1}\right] \cosh \nu \theta_{0}+\left[\frac{\hat{k}_{e}}{2}-\frac{2 i \lambda \hat{\omega} a_{s}}{\hat{k}_{e} U_{2} \hat{\beta}^{2}} c_{\nu}^{(3)}+\hat{\xi}\left(c_{\nu+1}^{(3)}-c_{\nu-1}^{(3)}\left(1+\delta_{\nu-1,0}\right)\right)\right.\right. \\
& \left.\left.-i \widetilde{\beta} c_{\nu}^{(2)}\right]\right\}+\sin (\beta-\theta)\left\{\frac{\hat{k}_{e} \hat{\beta}}{2}\left(a_{\nu-1}+a_{\nu+1}\right) \sinh \left(\nu \theta_{0}\right)+i \widetilde{\beta} c_{\nu}^{(1)}+\frac{i \hat{\omega} a_{s}}{U_{2}} c_{\nu}^{(3)}\right\}-\left(\bar{u}_{1}-\bar{u}_{2}\right) \sin (\beta-\theta) \frac{\hat{k}_{e}}{2}\left\{-\frac{2 i U_{2} \hat{\omega} \lambda}{\hat{k}_{e} \hat{\beta}^{2} a_{s}} d_{\nu}\right. \\
& \left.+\hat{\xi}\left(d_{\nu+1}-d_{\nu-1}\left(1+\delta_{\nu-1,0}\right)\right)\right\}=R_{\nu}^{(1)} \\
& \left\{\begin{array}{c}
A_{1} \\
B_{1}
\end{array}\right\}\left\{\operatorname { s i n } ( \beta - \theta ) \left[-\frac{\hat{k}_{e}}{2}\left[\frac{2 i \hat{\omega} U_{2}}{\hat{k}_{e} \hat{\beta}^{2} a_{s}} a_{\nu}+a_{\nu-1}\left(1+\delta_{\nu-1,0}\right)-a_{\nu+1}\right] \cosh \nu \theta_{0}+\left[\frac{\hat{k}_{e}}{2}\left(-\frac{2 i \lambda \hat{\omega} a_{s}}{\hat{k}_{e} U_{2} \hat{\beta}^{2}} c_{\nu}^{(3)}+\hat{\xi}\left(c_{\nu+1}^{(3)}-c_{\nu-1}^{(3)}\left(1+\delta_{\nu-1,0}\right)\right)\right)\right.\right.\right.
\end{aligned}
$$

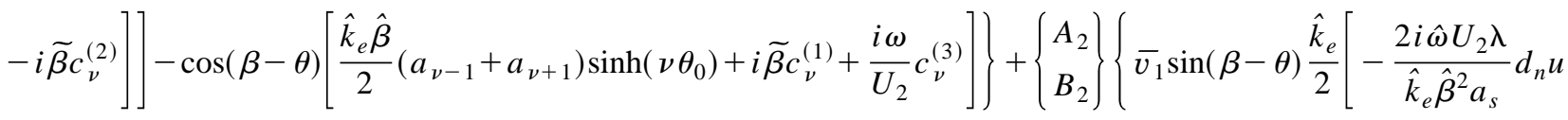

$$
\begin{aligned}
& \left.\left.+\hat{\xi}\left(d_{\nu+1}-d_{\nu-1}\left(1+\delta_{\nu-1,0}\right)\right)\right]+i \omega d_{\nu}\right\}+\left\{\begin{array}{c}
A_{3} \\
B_{3}
\end{array}\right\}\left\{\frac{\hat{k}_{e}}{2}\left\{\frac{2 i \hat{\omega}}{\hat{k}_{e} \hat{\beta}^{2}} a_{\nu}+\frac{U_{2}}{a_{s}} a_{\nu-1}\left(1+\delta_{\nu-1,0}\right)-\frac{U_{2}}{a_{s}} a_{\nu+1}\right\} \cosh \left(\nu \theta_{0}\right)\right\}=\left\{\begin{array}{c}
R_{\nu}^{(2)} \\
R_{\nu}^{(3)}
\end{array}\right\},
\end{aligned}
$$

and

$$
\begin{gathered}
i \alpha_{3} \cosh \left(\nu \theta_{0}\right) a_{\nu}-\frac{i \omega}{U_{2}} c_{\nu}^{(2)}-\frac{\hat{k}_{e}}{2}\left(-\frac{2 i \lambda \hat{\omega} a_{s}}{\hat{k}_{e} U_{2} \hat{\beta}^{2}} c_{\nu}^{(1)}+\hat{\xi}\left(c_{\nu+1}^{(1)}\right.\right. \\
\left.\left.-c_{\nu-1}^{(1)}\right)\left(1+\delta_{\nu-1,0}\right)\right)-i \alpha_{3}\left(\bar{u}_{1}-\bar{u}_{2}\right) d_{\nu}=R_{\nu}^{(4)}
\end{gathered}
$$

These equations must also be augmented with the condition arising from the solenoidal condition on the vector potential, namely,

$$
\begin{aligned}
& -\frac{i \omega}{U_{2}} c_{\nu}^{(1)}+\frac{\hat{k}_{e}}{2}\left(-\frac{2 i \lambda \hat{\omega} a_{s}}{k_{e} U_{2} \hat{\beta}^{2}} c_{\nu}^{(2)}+\hat{\xi}\left(c_{\nu+1}^{(2)}-c_{\nu-1}^{(2)}\right)\right. \\
& \left.\times\left(1+\delta_{\nu-1,0}\right)\right)+i \alpha_{3} c_{\nu}^{(3)}=0 .
\end{aligned}
$$

Terms used in the above are

$$
\tanh \theta_{0}=\hat{\beta} \tan (\beta-\theta),
$$

$$
A_{1}=\frac{1}{2} \text {, }
$$




$$
\begin{aligned}
& A_{2}=\frac{1}{2}\left(1-\frac{1}{\rho_{2}}\right), \\
& A_{3}=\frac{a_{s}}{2 \overline{u_{2}}}, \\
& B_{1}=1+\frac{a_{s}^{2}}{(\gamma-1) \bar{u}_{2}^{2}}, \\
& B_{2}=-\left[\frac{a_{s}^{2}}{(\gamma-1) \bar{u}_{2}^{2}}-\frac{\overline{u_{1}}}{\overline{u_{2}}}\right]\left(1-\frac{1}{\rho_{2}}\right), \\
& B_{3}=\frac{\gamma a_{s}}{(\gamma-1) \bar{u}_{2}} .
\end{aligned}
$$

The $R_{\nu}^{(n)}$ as in Duck et al., ${ }^{11}$ are to be determined from the freestream conditions. In particular in order to write the exponential terms, arising from the upstream solution, in terms of Bessel functions the following is particularly useful:

$\exp \left[i\left(\alpha_{1} \cot \beta+\alpha_{2}\right)(\cos \theta+\lambda \sin \theta) y_{2}\right]$

$$
\begin{aligned}
= & \exp \left(-i \frac{U_{2} \omega \lambda}{U_{2}^{2}-a_{s}^{2}} y_{2}\right) e^{i \sin \widetilde{\theta} \hat{k}_{e} \hat{\xi}_{2}} \\
= & \exp \left(-i \frac{U_{2} \omega \lambda}{U_{2}^{2}-a_{s}^{2}} y_{2}\right) \sum_{\nu=0}^{\infty} \frac{e^{i \nu \widetilde{\theta}}+(-1)^{\nu} e^{-i \nu \widetilde{\theta}}}{1+\delta_{\nu, 0}} \\
& \times J_{\nu}\left(\hat{k}_{e} \hat{\xi} y_{2}\right),
\end{aligned}
$$

where

$$
\widetilde{\theta}=\sin ^{-1}\left\{\frac{\left(\alpha_{1} \cot \beta+\alpha_{2}\right)(\cos \theta+\lambda \sin \theta)+\frac{U_{2} \omega \lambda}{U_{2}^{2}-a_{s}^{2}}}{\hat{k}_{e} \hat{\xi}}\right\} .
$$

Other details are routine, and omitted for reasons of brevity.

The combined system (62)-(65) then represents a closed (recursive-type) system determining the $a_{\nu+1}, c_{\nu+1}^{(n)}, d_{\nu+1}$.

\section{SOME NUMERICAL RESULTS}

The first set of data we present is for the particular case $M_{1}=5, \theta=25^{\circ}$ (weak shock solution), for the particular case of a slow acoustic mode with $\alpha_{1}=1, \alpha_{2}=0$, and $\alpha_{3}=1$. Fig. 1(a) shows results for the perturbation pressure on the wall $\left(y_{2}=0\right)$. Here, and in all cases the solidus denotes the real part of a function, the dashed line the imaginary part. This figure presents a picture reminiscent of results found in a number of corresponding two-dimensional cases by Duck et al., ${ }^{11}$ namely, that of an oscillatory-type nature downstream. Fig. 1(b) shows the corresponding perturbation shock location; this too takes on a similarly oscillatory nature. Figs. 2(a) and 2(b) show the corresponding results for the fast mode case (other data remaining the same as for Fig. 1). These figures are to be compared with Fig. 2 and Fig. 3 of Duck et al. ${ }^{11}$ where the same conditions apply except that $\alpha_{3}=0$ in those graphs. The difference between the twodimensional results and the three-dimensional results depends on whether or not the disturbance produces an acoustic wave behind the shock that intersects with the surface. In
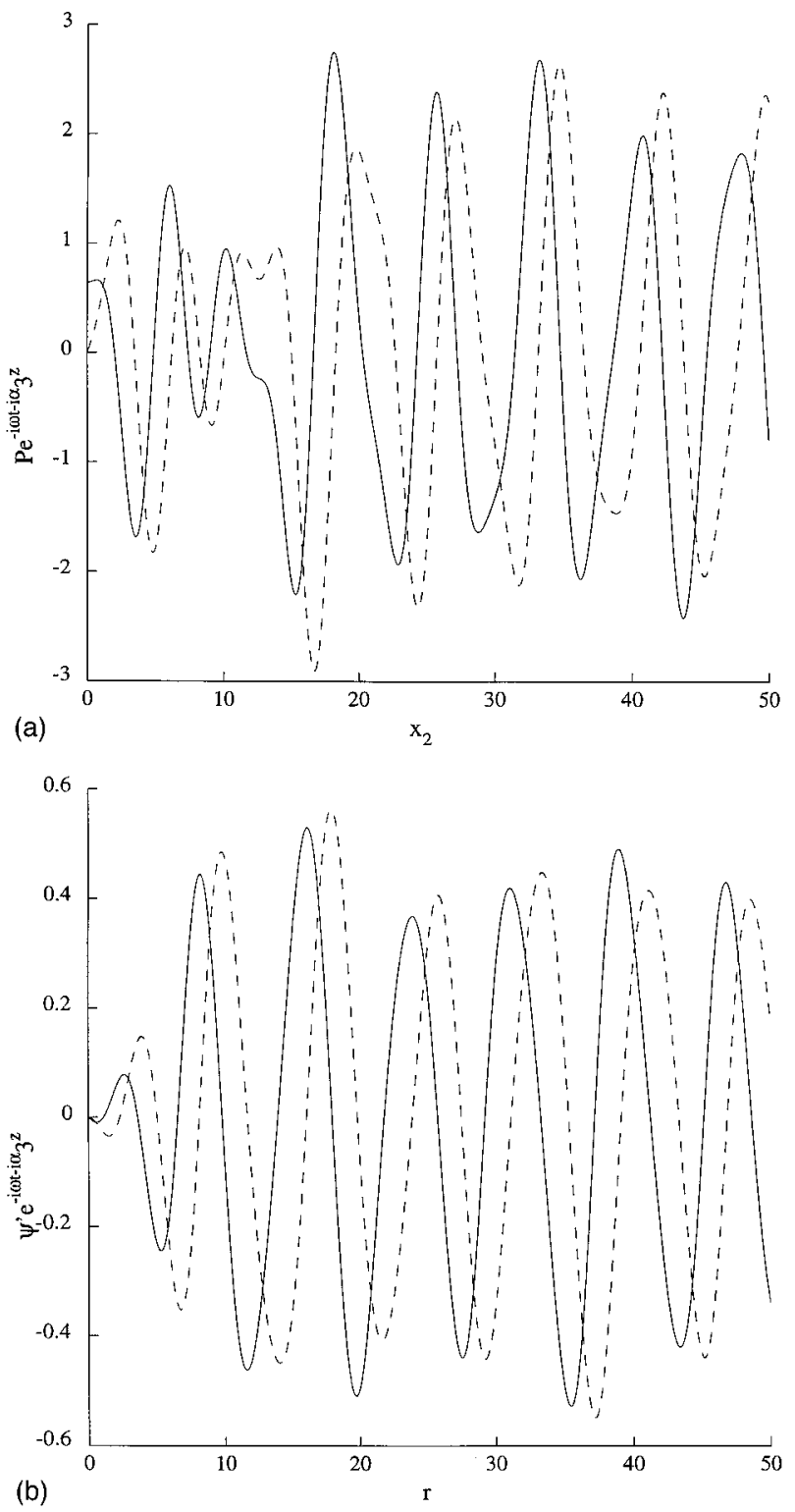

FIG. 1. (a) Wall pressure perturbations, $M_{1}=5, \theta=25^{\circ}$ (weak-shock solution), slow acoustic mode, $\alpha_{1}=1, \alpha_{2}=0, \alpha_{3}=1$. (b) Shock location perturbations, $M_{1}=5, \theta=25^{\circ}$ (weak-shock solution), slow acoustic mode, $\alpha_{1}=1, \alpha_{2}=0, \alpha_{3}=1$.

response to this particular slow mode, the pressure response at the surface for the two-dimensional disturbance decays algebraically while the shock position shows a characteristic single wavelength response in the far-field limit. On the other hand, the surface pressure response to the threedimensional slow-mode disturbance does not decay and shows a multi-wavelength pattern as is to be expected if a decomposition of the far field behaviour applies to the threedimensional case as it does for the two-dimensional case. The shock position shows a two-wavelength structure as would be expected by the primary acoustic disturbance reflecting off the wedge surface and interacting with the shock from behind. There is very little difference between the responses to a two-dimensional fast-mode disturbance and a three-dimensional fast-mode disturbance. A search in parameter space would lead to the same conclusion as in the two- 

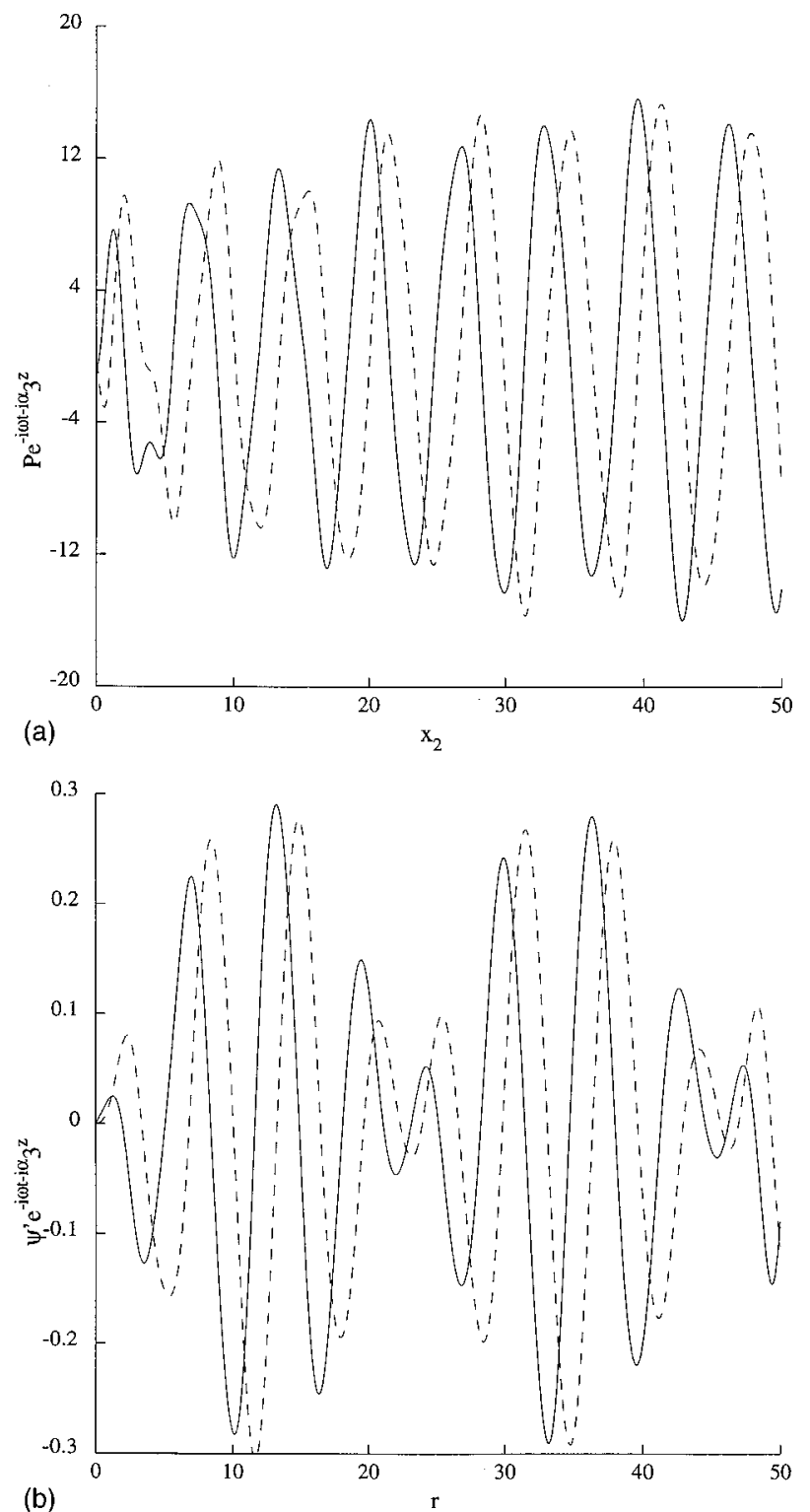

FIG. 2. (a) Wall pressure perturbations, $M_{1}=5, \theta=25^{\circ}$ (weak-shock solution), fast acoustic mode, $\alpha_{1}=1, \alpha_{2}=0, \alpha_{3}=1$. Shock location perturbations, $M_{1}=5, \theta=25^{\circ}$ (weak-shock solution), fast acoustic mode, $\alpha_{1}=1$, $\alpha_{2}=0, \alpha_{3}=1$.

dimensional case: either the surface pressure has a nondecaying component and an algebraically decaying component or it has only an algebraically decaying component. This is significant in that the solution in the absence of a wedge indicates that the choices are between only a nondecaying response and an exponentially decaying response (see Hussaini et al., ${ }^{15}$ Jackson et al., ${ }^{16}$ Lasseigne and Hussaini ${ }^{17}$ ). This issue was pursued in detail in Duck et al. ${ }^{11}$ where it is determined that the condition that the shock re- main attached to the apex of the wedge is the source for the algebraically decaying portion of the response - the apex is in effect a point source for additional acoustic waves. The interaction of these algebraically decaying acoustic waves with the shock in turn produces an algebraically decaying portion in both the entropy and vorticity modes. Another reason that it is important to determine that the pressure has only a decaying or sustained oscillatory behaviour downstream is that, as mentioned in the analysis of the response to the two-dimensional disturbances, the potential functions have components of exponential growth in them. The calculation of the physical quantities of pressure, velocity and vorticity are therefore dependent on some fortuitous cancellations, or they themselves would be exponentially growing. In the following section we investigate the downstream behaviour of the three-dimensional perturbation solution in some detail.

\section{THE FAR DOWNSTREAM BEHAVIOUR/STABILITY PROBLEM}

The results of the previous section show that a general growth in physical quantities, as $x_{2}$ increases does not occur even though the potential functions have an exponentially growing component in them. This is similar to the twodimensional results as considered by Duck et al., ${ }^{11}$ in which it was shown by examining the recurrence relations that only decaying or sustained physical solution oscillations were possible. The same type of analysis is possible for the threedimensional case, but indeed has its own peculiarities as will be seen.

In order to analyze the $x_{2} \rightarrow \infty$ behaviour, we consider the homogeneous solution of the system (62)-(65), and consider the limit as $\nu \rightarrow \infty$. In particular, we write

$c_{\nu}^{(n)} \approx C_{0}^{(n)} K^{\nu}, \quad d_{\nu} \approx D_{0} K^{\nu}, \quad$ and $a_{\nu} \approx A_{0} K^{\nu} e^{-\nu \theta_{0}}$,

where $K$ is an eigenvalue whose precise value (including location in complex space) serves to determine the ultimate downstream behaviour of the disturbance. If $|K|>1$, then using the generating function for Bessel functions, we have (for example)

$$
\begin{aligned}
\sum_{\nu=1}^{\infty} d_{\nu} J_{\nu}(z) & \approx \sum_{\nu=-\infty}^{\infty} D_{0} K^{\nu} J_{\nu}(z) \\
& \approx D_{0} e^{\frac{1}{2}\left(K-\frac{1}{K}\right) z} \quad \text { as }|z| \rightarrow \infty .
\end{aligned}
$$

Thus exponential growth occurs as $|z| \rightarrow \infty$ if $\mathfrak{R}\{z(K-1 / K)\}>0$. If we substitute (76) into (62)-(65), allow $\nu \rightarrow \infty$, and discard the inhomogeneous terms, then we obtain the following five linear homogeneous equations for the coefficients $A_{0}, C_{0}^{(n)}$ and $D_{0}$ :

$$
\frac{1}{2} i \widetilde{\beta} A_{0}-\frac{i \omega}{U_{2}} C_{0}^{(2)}-\hat{k}_{e}\left[-\frac{i \lambda \hat{\omega} a_{s}}{\hat{k}_{e} U_{2} \hat{\beta}^{2}}+\frac{1}{2} \hat{\xi}\left(K-\frac{1}{K}\right)\right] C_{0}^{(1)}-i \widetilde{\beta}\left(\overline{u_{1}}-\bar{u}_{2}\right) D_{0}=0,
$$




$$
\begin{aligned}
& -\frac{i \omega}{U_{2}} C_{0}^{(1)}+\hat{k}_{e}\left[-\frac{i \lambda \hat{\omega} a_{s}}{\hat{k}_{e} U_{2} \hat{\beta}^{2}}+\frac{1}{2} \hat{\xi}\left(K-\frac{1}{K}\right)\right] C_{0}^{(2)}+i \widetilde{\beta} C_{0}^{(3)}=0, \\
& \cos (\beta-\theta)\left\{-\frac{\hat{k}_{e}}{4}\left[\frac{2 i \hat{\omega} U_{2}}{\hat{k}_{e} \hat{\beta}^{2} a_{s}}+\frac{e^{\theta_{0}}}{K}-e^{-\theta_{0}} K\right] A_{0}+\hat{k}_{e}\left[-\frac{i \lambda \hat{\omega} a_{s}}{\hat{k}_{e} U_{2} \hat{\beta}^{2}}+\frac{1}{2} \hat{\xi}\left(K-\frac{1}{K}\right)\right] C_{0}^{(3)}-i \widetilde{\beta} C_{0}^{(2)}\right\} \\
& +\sin (\beta-\theta)\left\{\frac{\hat{k}_{e} \hat{\beta}}{4}\left(\frac{e^{\theta_{0}}}{K}+e^{-\theta_{0}} K\right) A_{0}+i \widetilde{\beta} C_{0}^{(1)}+\frac{i \omega}{U_{2}} C_{0}^{(3)}\right\}-\left(\overline{u_{1}}-\overline{u_{2}}\right) \sin (\beta-\theta) \hat{k}_{e}\left\{-\frac{i U_{2} \hat{\omega} \lambda}{\hat{k}_{e} \hat{\beta}^{2} a_{s}}+\frac{\hat{\xi}}{2}\left(K-\frac{1}{K}\right)\right\} D_{0}=0, \\
& \left\{\begin{array}{l}
A_{1} \\
B_{1}
\end{array}\right\}\left\{\sin (\beta-\theta)\left[-\frac{\hat{k}_{e}}{4}\left(\frac{2 i \hat{\omega} U_{2}}{\hat{k}_{e} \hat{\beta}^{2} a_{s}}+\frac{e^{\theta_{0}}}{K}-e^{-\theta_{0}} K\right) A_{0}+\hat{k}_{e}\left[-\frac{i \lambda \hat{\omega} a_{s}}{\hat{k}_{e} U_{2} \hat{\beta}^{2}}+\frac{1}{2} \hat{\xi}\left(K-\frac{1}{K}\right)\right] C_{0}^{(3)}-i \widetilde{\beta} C_{0}^{(2)}\right]\right. \\
& -\cos (\beta-\theta)\left[\frac{\hat{k}_{e} \hat{\beta}}{2}\left(\frac{e^{\theta_{0}}}{K}+e^{-\theta_{0} K}\right) A_{0}+i \widetilde{\beta} C_{0}^{(1)}+\frac{i \omega}{U_{2}} C_{0}^{(3)}+\left\{\begin{array}{c}
A_{2} \\
B_{2}
\end{array}\right\}\left\{\overline{v_{1}} \sin \left(\beta-\theta_{0}\right)\left[\hat{k}_{e}\left(-\frac{i \hat{\omega} U_{2} \lambda}{\hat{k}_{e} \hat{\beta}^{2} a_{s}}+\frac{\hat{\xi}}{2}\left(K-\frac{1}{K}\right)\right)+i \omega\right] D_{0}\right\}\right. \\
& +\left\{\begin{array}{c}
A_{3} \\
B_{3}
\end{array}\right\}\left\{\frac{\hat{k}_{e}}{2}\left(\frac{2 i \hat{\omega}}{\hat{k}_{e} \hat{\beta}^{2}}+\frac{U_{2}}{a_{s}} \frac{e^{\theta_{0}}}{K}-\frac{U_{2}}{a_{s}} e^{-\theta_{0} K}\right) A_{0}\right\}=0 .
\end{aligned}
$$

This equation is nonlinear in $K$ but can be converted to a tenth-order linear, generalized eigenvalue problem, which may be solved using the $Q Z$ algorithm by using the additional variables

$$
\hat{A}_{0}=K A_{0}, \quad \hat{C}_{0}^{(n)}=K \hat{C}_{0}^{(n)}, \quad \hat{D}_{0}=K D_{0} .
$$

It was shown by Duck et al. ${ }^{11}$ that in the two-dimensional case, for situations in which the downstream flow was supersonic, there were four imaginary eigenvalues (all with $|K|<1$ ), and two complex eigenvalues given by

$$
K=\frac{e^{\theta_{0}}}{U_{2}}\left\{i a_{s} \pm\left[U_{2}^{2}-a_{s}^{2}\right]^{1 / 2}\right\} .
$$

However, although these complex eigenvalues are such that $|K|>1$, in both cases $D_{0}=0$, and due to some "fortuitous" cancellations, all physical quantities remain bounded as $x_{2} \rightarrow \infty$ even though it is determined that the potential functions themselves grow exponentially.

In the three-dimensional case, we may expect, ten eigenvalues instead of the six in the two-dimensional case. However, it turns out generally that there are just eight distinct eigenvalues (two of the eigenvalues being double eigenvalues). Generally we find (i) four imaginary eigenvalues, (ii) one complex conjugate pair of double eigenvalues of the form $\pm K_{r}+i K_{i}$, and (iii) one complex conjugate pair of eigenvalues also of the form $\pm K_{r}+i K_{i}$.

For $\theta=25^{\circ}, M_{1}=5, \alpha_{1}=1$ and $\alpha_{2}=0$, the four imaginary eigenvalues have magnitude less than unity even as $\alpha_{3}$ increases from zero. The eigensolutions associated with these eigenvalues do not contribute to any growth in any of the quantities calculated. Most interesting, however, is the variation of these four eigenvalues as the wedge angle is increased. All four eigenvalues approach $K=i$ at the maximum wedge angle.

The (imaginary part of the) eigenvalues described by (i) are shown in Fig. 3(a), whilst the real and imaginary parts of the eigenvalues described by (ii) and (iii) are shown in Figs. 3(b) and 3(c), respectively. Here, we have just shown the eigenvalues with the positive value of $K_{r}$ (the other eigenvalue, corresponding to $-K_{r}+i K_{i}$ may obviously be simply deduced). We next consider the effect of varying $\theta$, for the parameters $M_{1}=5, \alpha_{1}=1, \alpha_{2}=0$, and $\alpha_{3}=1$. The variation of $K_{i}$ of family (i) is shown in Fig. 4(a), and the variation of the real and imaginary parts of eigenvalues described by (ii) and (iii) is shown in Figs. 4(b) and 4(c), respectively. Again, just the positive values of $K_{r}$ are shown. It is seen that the double eigenvalues described by (ii) are the only ones with $|K|>1$ and therefore lead to potential exponential growth. It turns out that an analytic description of the eigenvalues described by (ii) is possible. The result is

$$
K=\frac{e^{\theta_{0}}}{U_{2}}\left\{\frac{i \hat{k} a_{s}}{\hat{k}_{e}} \pm\left[U_{2}^{2}-\frac{a_{s}^{2} \hat{k}^{2}}{\hat{k}_{e}^{2}}\right]^{1 / 2}\right\} .
$$

It is also possible to obtain analytic results for one set of eigen-coefficients corresponding to these eigenvalues, namely

$$
C_{0}^{(1)}=\frac{-\frac{i A_{0} U_{2}}{2}\left\{-\frac{\alpha_{3} \hat{k}_{e} \hat{\beta}}{2}\left(K e^{-\theta_{0}}+\frac{e^{\theta_{0}}}{K}\right)+\alpha_{3}\left[\frac{\hat{k}_{e} \hat{\xi}}{2}\left(K-\frac{1}{K}\right)-\frac{\lambda i \hat{k} a_{s}}{U_{2}}\right]^{2}\right\}}{\alpha_{3}^{2} U_{2}+\frac{\omega^{2}}{U_{2}}-U_{2}\left[\frac{i \lambda \hat{k} a_{s}}{U_{2}}-\frac{\hat{k}_{e} \hat{\xi}}{2}\left(K-\frac{1}{K}\right)\right]^{2}}
$$



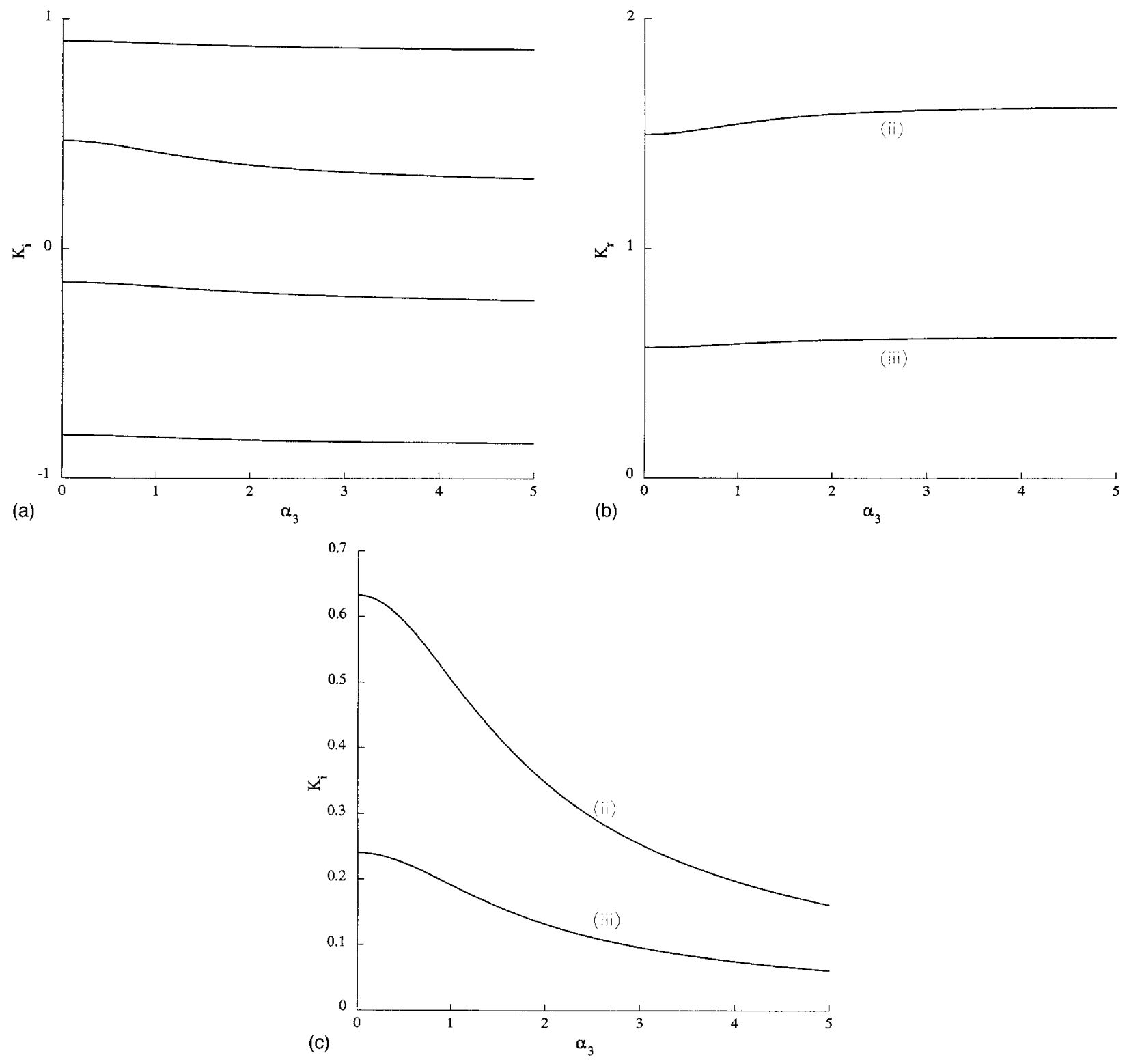

FIG. 3. (a) Variation of $K_{i}$ of the eigenvalue family (i) with $\alpha_{2}$, for $\theta=25^{\circ}, M_{1}=5$ (weak-shock solution), $\alpha_{1}=1, \alpha_{2}=0$. (b) Variation of $K_{i}$ of the eigenvalue family (ii) and (iii) with $\alpha_{2}$, for $\theta=25^{\circ}, M_{1}=5$ (weak-shock solution), $\alpha_{1}=1, \alpha_{2}=0$. (c) Variation of $K_{r}$ of the eigenvalue family (ii) and (iii) with $\alpha_{2}$, for $\theta=25^{\circ}, M_{1}=5$ (weak-shock solution), $\alpha_{1}=1, \alpha_{2}=0$.

$$
\begin{aligned}
& C_{0}^{(2)}=-\frac{i U_{2}}{\omega}\left\{\frac{1}{2} i \alpha_{3} A_{0}+C_{0}^{(1)}\left[\frac{i \lambda \hat{k} a_{s}}{U_{2}}-\frac{\hat{k}_{e} \hat{\xi}}{2}\left(K-\frac{1}{K}\right)\right]\right\}, \\
& C_{0}^{(3)}=\frac{i U_{2}}{\omega}\left\{\frac{\hat{k}_{e} \hat{\beta}}{4}\left(K e^{-\theta_{0}}+\frac{e^{\theta_{0}}}{K}\right) A_{0}+i \alpha_{3} C_{0}^{(1)}\right\}, \\
& D_{0}=0 .
\end{aligned}
$$

The above solution readily reduces to the two-dimensional solution in the limit $\alpha_{3} \rightarrow 0$. Some understanding of the origin of this other family of coefficients for this eigenvalue, and also of the additional complex eigenvalue (iii) may be made by considering the two-dimensional limit, $\alpha_{3} \rightarrow 0$ and seeking a solution to the system with $A_{0}=D_{0}=C_{0}^{(3)}=0$. After some algebra, we find the following (four) eigenvalues:

$$
K=\frac{\lambda i \hat{k} a_{s}}{\hat{k}_{e} \hat{\xi} U_{2}} \pm\left\{\left[-\frac{\lambda i \hat{k} a_{s}}{\hat{k}_{e} \hat{\xi} U_{2}} \pm \frac{\omega}{U_{2} \hat{k}_{e} \hat{\xi}}\right]^{2}-1\right\}^{1 / 2},
$$

together with

$$
C_{0}^{(1)}=-\frac{U_{2} \hat{k}_{e} \hat{\xi} i}{2 \omega} C_{0}^{(2)}\left\{K-\frac{1}{K}-\frac{2 \lambda i \hat{k} a_{s}}{U_{2} \hat{k}_{e} \hat{\xi}}\right\} .
$$

Two of the above values for $K$ correspond to (83), and this partly explains the origin of the double eigenvalues. As $\alpha_{3}$ increases from zero, it is expected that $A_{0}, D_{0}$, and $C_{0}^{(3)}$ will 


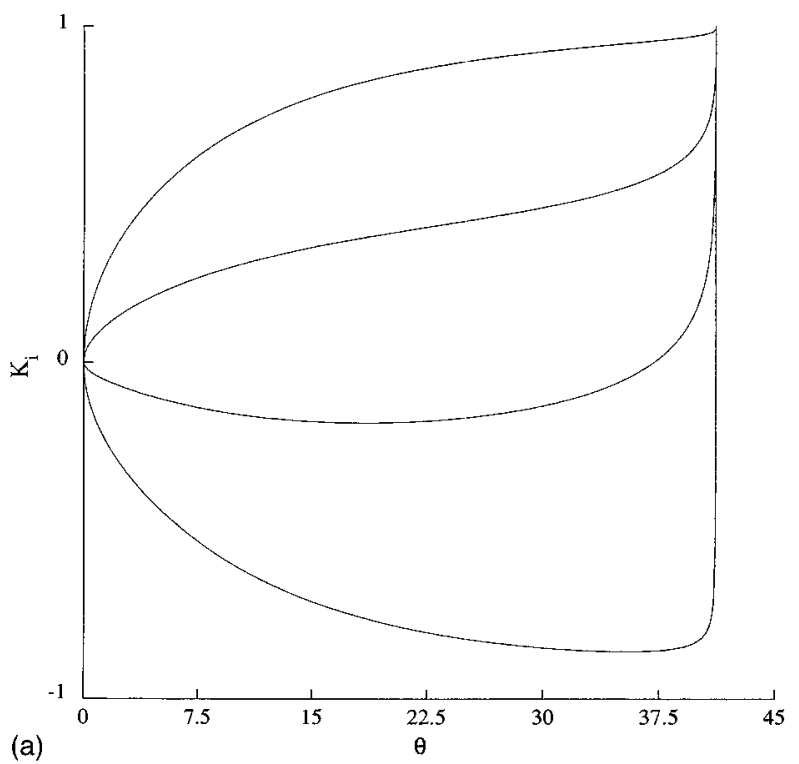

(a)

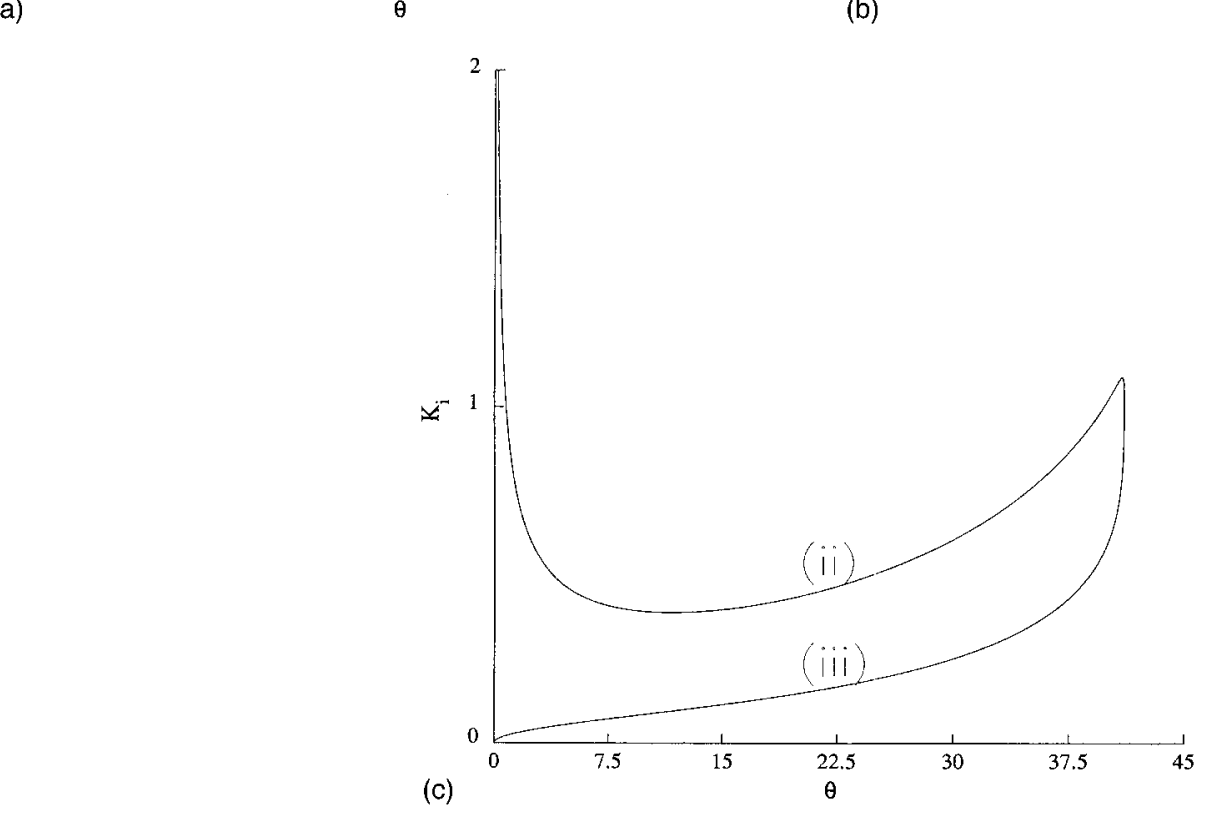

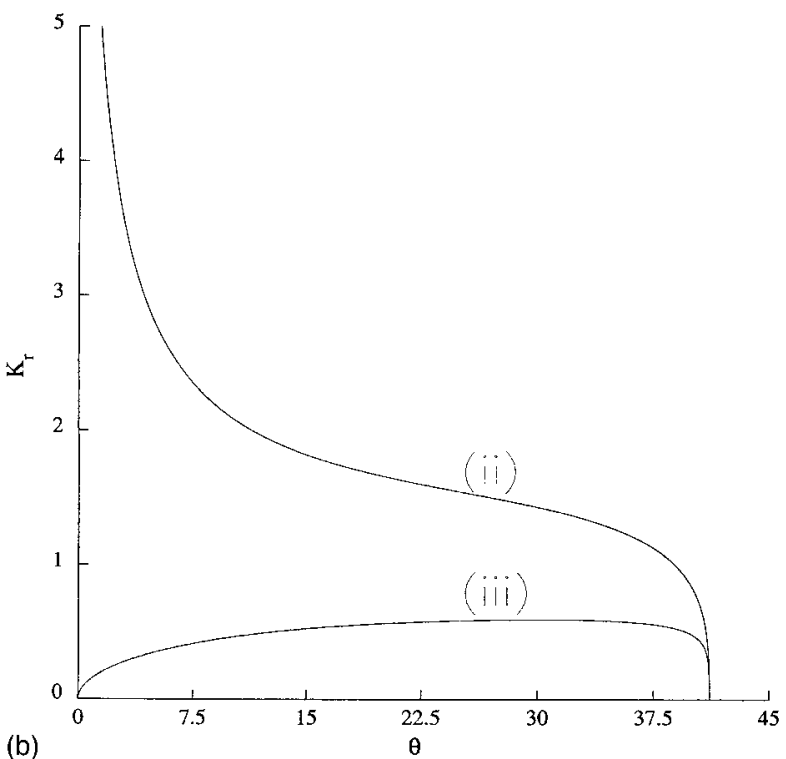

(b)

\section{.}

FIG. 4. (a) Variation of $K_{i}$ of the eigenvalue family (i) with $\theta$, for $M_{1}=5$ (weak-shock solution), $\alpha_{1}=1, \alpha_{2}=0, \alpha_{3}=1$. (b) Variation of $K_{r}$ of the eigenvalue family (ii) and (iii) with $\theta$, for $M_{1}=5$ (weak-shock solution), $\alpha_{1}=1, \alpha_{2}=0, \alpha_{3}=1$. (c) Variation of $K_{i}$ of the eigenvalue family (ii) and (iii) with $\theta$, for $M_{1}=5$ (weak-shock solution), $\alpha_{1}=1, \alpha_{2}=0, \alpha_{3}=1$.

no longer be zero for this branch. The other pair of eigenvalues correspond to the eigenvalues described by (iii).

Again it is important to note is that with the exception of the eigenvalues (ii), all eigenvalues of $K$ correspond to $|K|<1$, and thus are of limited physical significance. Interestingly at the maximum value of $\theta$ (corresponding to the maximum wedge angle for attached flow at a particular freestream Mach number), all eigenvalues take on the same value, $K=i$. Turning now to the effects of the eigenvalues described by (ii), again, as in the two-dimensional case, it turns out that although seemingly these eigensolutions correspond to unstable (growing) downstream solutions, in fact the evaluation of physical quantities leads to solutions downstream which remain bounded. This is best illustrated by defining the following set of functions:

$$
\begin{aligned}
& \widetilde{E}^{(n)}= C_{0}^{(n)} e^{i \omega t-\frac{i \omega x_{2}}{U_{2}}-\frac{i \omega \lambda y_{2} a_{s}^{2}}{U_{2}\left(U_{2}^{2}-a_{s}^{2}\right)}+i \alpha_{3} z} \\
& \times \sum_{\nu=-\infty}^{\infty} K^{\nu} J_{\nu}\left(\hat{k}_{e} \hat{\xi} y_{2}\right), \\
& \widetilde{\phi}=A_{0} e^{i \omega t-} \frac{i \omega U_{2} x_{2}}{U_{2}^{2}-a_{s}^{2}}+i \alpha_{3} z \sum_{\nu=-\infty}^{\infty}\left\{K e^{\theta_{2}-\theta_{0}}\right\}^{\nu} J_{\nu}\left(\hat{k}_{e} r\right),
\end{aligned}
$$

where $K$ is defined by (83) and we write $\widetilde{\mathbf{E}}=\left(\widetilde{E}^{(1)}, \widetilde{E}^{(2)}, \widetilde{E}^{(3)}\right)$. It would be expected that $\widetilde{E}$ and $\widetilde{\phi}$ determine the far-downstream behaviour of the flow which grows downstream. However, inspection of the solution above, in the cases considered, leads to the conclusion that 


$$
\boldsymbol{\nabla} \widetilde{\phi}=-\nabla \wedge \widetilde{\mathbf{E}}
$$

This then leads to all velocity components (and hence all physical quantities) remaining bounded downstream. Numerically, it is determined that all physical quantities remained bounded no matter what the parameters selected for the calculation, thus the above must hold for all eigensolutions with $|K|>1$.

In summary, therefore, we see that provided the flow downstream is supersonic then disturbances will not grow downstream. This boundary of downstream behaviours is independent of the crossflow wave number $\alpha_{3}$, and occurs along the "line" where $\hat{\beta}=0$ (where $\hat{k}$ and $\hat{k}_{e}$ simultaneously become singular, and also change from being real to imaginary quantities). The result is that all of the Bessel functions in the series (39)-(40) will grow downstream if the downstream flow is subsonic (although we note the comments of Salas and Morgan ${ }^{10}$ stating that strong shock solutions could perhaps be stable under a different set of boundary conditions).

We now address the nature of the downstream response to sustained excitation (i.e., acoustic waves, entropy waves, or vorticity waves). Arguments similar to those employed by Duck et al. ${ }^{11}$ are again appropriate. The frequency of the disturbance downstream of the shock is determined by the frequency of the disturbance upstream of the shock and both wave numbers of the disturbance tangential to the shock are also fixed by the deflection of the shock produced by the upstream disturbance. There is a finite range of tangential wave numbers that exclude the existence of plane acoustic waves which propagate downstream. If the tangential wave numbers lie outside of this range, then a plane wave propagates at an angle $\theta_{p}$ to the shock. In the case of the tangential wave numbers being within the excluded range, an acoustic field is generated that decays algebraically rather than exponentially as would be the case in the absence of the wedge. If the tangential wave number is outside of the excluded range, then the solution in the presence of the wedge exhibits two types of behaviour, dependent upon the angle of propagation of an acoustic disturbance in the absence of the wedge. If the angle of propagation $\theta_{p}$ is greater than the angle between the shock and the wedge $\beta-\theta$, then the pressure disturbances generated at the shock intersect with and reflect from the wedge surface, leading to a non-decaying pressure field everywhere between the shock and the wedge surface, with a superimposed algebraically decaying pressure field owing to the requirement of an attached shock and zero velocity normal to the wedge surface. If the angle of propagation $\theta_{p}$ is less than the angle between the shock and the wedge surface, then the pressure field is divided into two regions by a ray emanating from the apex and parallel to the direction of propagation of the pressure disturbance in the absence of the wedge. Between the shock and this ray there is a nondecaying pressure field, with the aforementioned superimposed algebraically decaying component. Between the ray and the wedge surface the pressure field is algebraically decaying and there is no component with sustained oscillations. This was referred to as the shadow region by Duck et al. ${ }^{11}$

The various downstream limits are determined by the value of $\widetilde{\theta}$. If $\widetilde{\theta}$ is real, then the wave number of the imposed disturbances lies within the excluded range, and the pressure along any ray $\theta_{2}=$ constant decays algebraically For $\theta_{2}=\theta_{0}$ the solution does not decay leading to the appropriate oscillatory behaviour necessary to match conditions at the shock. If $\widetilde{\theta}$ is complex, then the condition

$$
\left|\frac{\left(\alpha_{1} \cot \beta+\alpha_{2}\right)(\cos \theta+\lambda \sin \theta)+\frac{U_{2} \omega \lambda}{U_{2}^{2}-a_{s}^{2}}}{\hat{k}_{e} \hat{\xi}}\right|>1,
$$

is satisfied. We also note that for equation (73) to be satisfied then $\sin \widetilde{\theta}$ must remain real, which requires that

$$
\widetilde{\theta}= \pm \pi / 2-i \widetilde{\theta}_{i},
$$

where

$$
\widetilde{\theta}_{i}=\cosh ^{-1}\left\{ \pm \frac{\left(\alpha_{1} \cot \beta+\alpha_{2}\right)(\cos \theta+\lambda \sin \theta)+\frac{U_{2} \omega \lambda}{U_{2}^{2}-a_{s}^{2}}}{\hat{k}_{e} \hat{\xi}}\right\} .
$$

$\widetilde{\theta}_{i}$ is related to the angle between the direction of the shock and the direction of the acoustic disturbance $\theta_{p}$ through $\tanh \widetilde{\theta_{i}}=\hat{\beta} \tan \theta_{p}$. If $\widetilde{\theta}_{i}$ is greater than $\theta_{0}$, then the generated or transmitted acoustic waves intersect the wedge surface. For $0<\widetilde{\theta}_{i}<\theta_{0}$, there are two regions: a region of sustained oscillatory behaviour of the pressure field near the shock when $\theta_{2}>\widetilde{\theta}_{i}$, and a region of algebraic decay of the pressure field near the wedge surface when $\theta_{2}<\widetilde{\theta}_{i}$.

These features are common to those found in the twodimensional case by Duck et al. ${ }^{11}$ Additionally, we can expect that the downstream (forced) behaviour will take the same form as in the two-dimensional case. Taking the wall pressure, for example, we may write the decomposition

$$
\begin{aligned}
e^{-i \omega t-i \widetilde{\beta} z} p= & P_{0} \exp \left(-\frac{i \omega U_{2} x_{2}}{U_{2}^{2}-a_{s}^{2}}+i \hat{k}_{e} \cosh \left(\widetilde{\theta}_{i}-\theta_{0}\right) x_{2}\right) \\
& +P_{1} x_{2}^{-1 / 2} \exp \left[-\left(\frac{i \omega x_{2}}{U_{2}-a_{s}}-i \frac{\pi}{4}\right)\right] \\
& +P_{2} x_{2}^{-1 / 2} \exp \left[-\left(\frac{i \omega x_{2}}{U_{2}+a_{s}}-i \frac{\pi}{4}\right)\right]+Q\left(x_{2}\right),
\end{aligned}
$$

i.e., a solution with an oscillatory component, two decaying acoustic waves, and a (faster) decaying component, $Q\left(x_{2}\right)$.

\section{CONCLUSIONS}

The interaction between a shock wave and threedimensional freestream disturbances has been considered, in particular the nature of the flow far downstream. The somewhat fortuitous cancelations that were found to occur in the analogous two-dimensional work (Duck et al. ${ }^{11}$ ), again intriguingly occur, yielding a non-growing physical solution downstream, thus confirming the stability of shocks with downstream supersonic flow, in line with the widely held 
belief regarding the stability of such shock waves. Our results also point to the "instability" of shocks with downstream subsonic flow, since in that case the argument of the Bessel functions in (39)-(42) becomes imaginary, and as a result exponential growth will be expected downstream. Finally we note that the location of the boundary between growing and non-growing downstream waves is unaffected by the crossflow wave number $\alpha_{3}$.

\section{ACKNOWLEDGMENTS}

The first author acknowledges the support of the Program in Computer Science and Engineering at Florida State University. Part of this work was carried out while the authors were in residence at the Institute for Computer Applications in Science and Engineering (ICASE), NASA Langley Research Center, Hampton, Virginia.

${ }^{1}$ F. K. Moore, "Unsteady oblique interaction of a shock wave with a plane disturbance,'” NACA Report No. 1165, 1954.

${ }^{2}$ H. S. Ribner, "Convection of a pattern of vorticity through a shock wave,' NASA Report No. 1164, 1954.

3 J. F. McKenzie and K. O. Westphal, "Interaction of linear waves with oblique shock waves,', Phys. Fluids. 11, 2310 (1968).

${ }^{4}$ G. F. Carrier, "The oscillatory wedge in a supersonic stream,' J. Aero. Sci. 16, 150 (1949).

5 M. D. Van Dyke, “'On supersonic flow past an oscillating wedge,', Q. Appl. Math. 11, 360 (1953).
${ }^{6}$ J. L. Levinson, 'L'etude de la stabilite des courant supersonique de gas en relation avec on double solution de la théorie des ondes de choc,' Prikl. Mat. Mekh. 9, 151 (1945).

${ }^{7}$ G. F. Carrier, "On the stability of the supersonic flow past a wedge,' Q. Appl. Math. 6, 356 (1949).

${ }^{8}$ L. F. Henderson and J. D. Atkinson, "Multi-valued solutions of steadystate supersonic flow,', Part I. Linear Analysis. J. Fluid Mech. 75, 751 (1970).

9 V. V. Rusanov and A. A. Sharakshange, "On the non-uniqueness of the solution of the problem on steady flow about the plane wedge and circular cone,' Comput. Fluids 7, 243 (1980).

${ }^{10}$ M. D. Salas and B. D. Morgan, "Stability of shock waves attached to wedges and cones,', AIAA J. 21, 1611 (1983).

${ }^{11}$ P. W. Duck, D. G. Lasseigne, and M. Y. Hussaini, "On the interaction between the shock wave attached to a wedge and freestream disturbances,', Theor. Comput. Fluid Dyn. 7, 119 (1995) (also ICASE Report No. 93-61).

${ }^{12}$ H. W. Liepmann and A. Roshko, Elements of Gas Dynamics (Wiley, New York, 1957).

${ }^{13}$ H. Poincaré, Théorie des Tourbillons (Carré, Paris, 1893).

${ }^{14}$ K. Aziz and J. D. Hellums, "Numerical solution of the three-dimensional equations of motion for laminar natural convection,'” Phys. Fluids 10, 314 (1967).

${ }^{15}$ M. Y. Hussaini, F. Collier, and D. M. Bushnell, "Turbulence alteration due to shock motion,' Proceedings of the IUTAM Symposium on Turbulent Shear-Layer/Shock Wave Interaction, edited by J. Delany (Springer, New York, 1985), p. 371.

${ }^{16}$ T. L. Jackson, A. K. Kapila, and M. Y. Hussaini, "Convection of a pattern of vorticity through a reacting shock wave,' Phys. Fluids A 2, 1260 (1990).

${ }^{17}$ D. G. Lasseigne and M. Y. Hussaini, "Interaction of disturbances with an oblique detonation wave attached to a wedge,', Phys. Fluids A 5, 1047 (1993). 